\title{
Asymptotic Behavior of a Discrete-Time Queue with Long Range Dependent Input
}

\author{
Tim Daniëls and Chris Blondia \\ Performance Analysis of Telecommunication Systems \\ Department of Mathematics and Computer Science \\ University of Antwerp \\ Universiteitsplein, 1, B-2610 Antwerp - Belgium \\ e-mail: \{tdaniels, blondia\}@uia.ua.ac.be
}

\begin{abstract}
-
In this paper we derive an expression for the asymptotics of the buffer length distribution of a discrete-time infinite capacity single server queue with deterministic service time and its input process belonging to a class of long range dependent discrete-time $\mathrm{M} / \mathrm{G} / \infty$ processes. This class of arrival process is defined as follows. At each time slot sequences of back-to-back customers are generated according to a Poisson distribution with parameter $\lambda$. The length of such a sequence is assumed to asymptotically behave like a Pareto distribution with parameter $s$, i.e. the probability that a sequence consists of $k$ customers is given by $c k^{-s}$ for $k \rightarrow \infty$, with $c>0$ and $2<s<3$. Due to the heavy tail of the members of this class of distributions, the presented class of $\mathrm{M} / \mathrm{G} / \infty$ processes has the long range dependence property (i.e. the autocorrelation function decays as a power of the lag time). We show that in this case the asymptotic behavior of the tail probabilities of the stationary distribution of the buffer occupancy is given by $\frac{\lambda c \rho^{s-2}}{(s-2)(s-1)(1-\rho)} n^{2-s}$ for $n \rightarrow \infty$, with $\rho$ representing the load of the system. This result is obtained using a generating function approach and the Tauberian theorem for power series. Furthermore, an application towards traffic management and simulation results are presented.

Keywords-M/G/ $\infty$ arrival process, long range dependence, buffer asymptotics
\end{abstract}

\section{INTRODUCTION}

In the past five years, traffic measurement studies on real packet networks, including Ethernet LAN traffic (see e.g. [1]), Variable Bit Rate video over the Asynchronous Transfer Mode (see e.g. [2]), etc., have shown that although the autocorrelations of the number of arrivals in a time interval decreases for higher lags, their cumulative effect diverges. This characteristic is in contrast with classical models used in teletraffic theory where the autocorrelation function exhibits an expontial decay. Traffic for which the autocorrelation function decays as a power of the lag time, is referred to as long range dependent (LRD), in contrast with the more classical short range dependent traffic (e.g. Markovian models).

There are several equivalent ways to express that a process $X_{k}$ has the LRD property. Assume that the autocorrelation function has a power-law decay, i.e. $C_{X}(k) \sim|k|^{-\beta}$. In the time domain, LRD implies nonsummable autocorrelations, i.e. $\sum_{k=1}^{\infty} C_{X}(k)=+\infty$, while in the frequency domain, LRD gives rise to a spectral density that obeys a power-law near the origin. The degree by which a process has the LRD property is expressed by means of the Hurst parameter. It is given by $H=1-\beta / 2$, where $\beta$ is the exponent of the power-law decay of the autocorrelation function and $H \in] 1 / 2,1]$.

The first author is aspirant with the Fonds voor Wetenschappelijk Onderzoek-Vlaanderen
Clearly this particular autocorrelation structure has a major impact on the performance of a queue to which this type of traffic is offered. Measurements, simulations and analytical studies have shown that the property of exponential decay rate of the tail of the queue length distribution observed for Markovian traffic, is not valid any longer when dealing with LRD input traffic. In this case, the queue length distribution may have a heavy tail which, when ignored, may lead to important underprovisioning of the required buffer size. Hence, in order to quantitatively investigate the influence of the LRD property on the queueing behavior, we need a traffic model which has the LRD property and we have to investigate the tail of the buffer of a single server deterministic queue whose input consists of this traffic. This is exactly the goal of this study.

In this paper, we consider the discrete-time $\mathrm{M} / \mathrm{G} / \infty$ input process. Intuitively, this process consists of sequences of back-toback customers. These sequences are generated at each time slot according to a Poisson distribution. The distribution of the number of customers in a train has a heavy tail, in order to ensure the LRD property. Back-to-back customers mean that the customers of a sequence arrive in consecutive time slots. This $\mathrm{M} / \mathrm{G} / \infty$ process naturally arises when considering an infinite superposition of identical on/off sources [3], [4], [5]. Furthermore, the M/G/ $\infty$ process studied in this paper is in several cases consistent with traffic measurements. See e.g. [6].

We study the tail probabilities of the stationary buffer distribution of a statistical multiplexer fed by a class of long range dependent discrete-time $\mathrm{M} / \mathrm{G} / \infty$ input processes. More precisely, we obtain the exact asymptotics describing the tail of the buffer distribution for the Pareto case. Several other papers, e.g. [7], [8], [9],[10], [3], address this topic, but most of them rely on large deviation techniques or focus on the continuous time case. Based on the approach presented in [11] and [12], we obtain the asymptotics of the buffer occupancy using generating functions and the elementary form of the Tauberian theorem for power series as presented in [13].

The paper is organized as follows. In the second section the input process is defined. In section III, an expression for the generating function of the buffer occupancy of the queueing system under study is derived. Section IV contains the main result of this paper: the asymptotics of the tail distribution of the buffer occupancy. Section V is devoted to the superposition of LRD and SRD traffic streams and to the consequences with respect to traffic management. In section VI several simulation results 
are presented. Some of them point out that the results obtained in section IV may be applicable in a more general setting than presented in this paper.

\section{THE DISCRETE-TIME M/G/ $\infty$ ARRIVAL PROCESS}

Since we are working in a discrete-time setting, time is divided in time slots. The discrete-time $\mathrm{M} / \mathrm{G} / \infty$ arrival process can be defined as follows. At the $k$-th timeslot a number $\gamma_{k}$ of new so-called sequences are generated according to a Poisson distribution with parameter $\lambda$. The random variables $\gamma_{k}$ are independent. Each sequence consists of a number of back-to-back customers (i.e. the customers belonging to a sequence arrive in consecutive time slots). Denote the random variable representing the length of a sequence, in number of customers, by $\tau_{A}$. Let the probability of a sequence of $k$ customers be $a_{k}=\mathbf{P}\left\{\tau_{A}=k\right\}$. The corresponding generating function is given by $A(z)=\sum_{k=1}^{\infty} a_{k} z^{k}$. Denote by $b_{k}$ the number of customers arriving at the $k$-th timeslot. The customer arrival rate is given by $\rho=\mathbf{E}\left[b_{k}\right]=\lambda \mathrm{E}\left[\tau_{A}\right]=\lambda A^{\prime}(1)$. In order for this $\mathrm{M} / \mathrm{G} / \infty$ process, which we denote by $\mathbf{A}$, to be long range dependent, it is required for $\tau_{A}$ to have a heavy tail. Hence we assume that $\tau_{A}$ is asymptotically distributed like a Pareto distribution with parameter $s$ with $2<s<3$, i.e.

$$
\mathbf{P}\left\{\tau_{A}=k\right\} \sim c k^{-s} \text { for } k \rightarrow \infty,
$$

with $c>0$. This distribution results in an arrival process with a finite mean and an infinite variance. As shown in e.g. [7] and [4],

$$
\operatorname{Cov}\left(b_{k}, b_{k+j}\right)=\lambda \sum_{n=j}^{\infty} \mathbf{P}\left\{\tau_{A}>n\right\},
$$

which implies the following power-law for the covariance function:

$$
\operatorname{Cov}\left(b_{k}, b_{k+j}\right) \sim \frac{\lambda c}{(s-1)(s-2)} j^{2-s}
$$

Hence the Hurst parameter $H=(4-s) / 2$.

In the next section we need a sequence of $\mathrm{M} / \mathrm{G} / \infty$ arrival processes $\mathbf{A}^{(n)}$ which converges weakly to $\mathbf{A}$. Consider therefor the sequence of random variables $\tau_{A^{(n)}}$ defined by

$$
\mathbf{P}\left\{\tau_{A^{(n)}}=i\right\}= \begin{cases}\mathbf{P}\left\{\tau_{A}=i\right\} & \text { if } i<n, \\ \mathbf{P}\left\{\tau_{A} \geqslant n\right\} & \text { if } i=n, \\ 0 & \text { if } i>n .\end{cases}
$$

The corresponding generating functions are denoted by $A^{(n)}(z)=\sum_{i=1}^{\infty} a_{i}^{(n)} z^{i}$, hence $A^{(1)}(z)=z, \ldots, A^{(n)}(z)=$ $a_{1} z+a_{2} z^{2}+\ldots+a_{n-1} z^{n-1}+\left(a_{n}+a_{n+1}+\ldots\right) z^{n}$. It is clear that the sequence $\tau_{A^{(n)}}$ converges weakly to $\tau_{A}$. Starting from $\tau_{A^{(n)}}$ instead of $\tau_{A}$ we can now use the method for constructing $\mathbf{A}$ to obtain $\mathbf{A}^{(n)}$. Hence $\left[\mathbf{A}^{(n)}\right]_{n}$ converges weakly to $\mathbf{A}$.

\section{THE GENERATING FUNCTION OF THE BUFFER OCCUPANCY DISTRIBUTION}

Consider a single server infinite capacity queue with deterministic service time equal to one time slot and arrival process the
M/G/ $\infty$ process A defined above. Denote this queueing system by QT. In order to derive an expression for the generating function of the buffer occupancy in this queueing system, we need to resort to a limit process in which two sequences of two different types of queueing systems are involved. The first sequence is denoted by $\left[\mathbf{Q T}{ }^{(n)}\right]_{n}$, the second one by $\left[\mathbf{Q B}{ }^{(n)}\right]_{n}$. Details about these sequences are given below.

\section{A. The sequence $\left[\mathbf{Q T} \mathbf{T}^{(n)}\right]_{n}$}

The queueing system $\mathbf{Q T}{ }^{(n)}$ is the single server infinite capacity queue with deterministic service time equal to one time slot and arrival process $\mathbf{A}^{(n)}$. Let $X_{S^{(n)}}$, resp. $X_{S}$ be the random variables representing the stationary buffer occupation distribution in the system $\mathbf{Q T}{ }^{(n)}$, resp. QT. Denote by $s_{k}^{(n)}$, resp. $s_{k}$, the probability that the buffer in the queueing system $\mathbf{Q T}{ }^{(n)}$, resp. QT, contains $k$ customers. Hence $S^{(n)}(z)=\sum_{k=0}^{\infty} s_{k}^{(n)} z^{k}$ and $S(z)=\sum_{k=0}^{\infty} s_{k} z^{k}$ are the generating functions corresponding to $X_{S(n)}$ and $X_{S}$ respectively.

By following the outline given in [11] or [12] we obtain

$$
\begin{aligned}
S^{(n)}(z)= & p_{0}^{(n)}(z-1) \sum_{i=1}^{n} \prod_{k=1}^{i} \frac{B\left(A_{k}(z)\right)}{z} \\
& +\left[\prod_{k=1}^{n} \frac{B\left(A_{k}(z)\right)}{z}\right] p_{0}^{(n)}(z-1) \frac{B\left(A_{n}(z)\right)}{z-B\left(A_{n}(z)\right)}
\end{aligned}
$$

with $B(z)=\exp [\lambda(z-1)]$ and $p_{0}^{(n)}=1-\rho^{(n)}=1-\lambda \mathbf{E}\left[\tau_{A_{n}}\right]$. Before we are able to determine an analytically tractable expression for $S(z)$, we need to introduce the queueing systems $\mathbf{Q B}^{(n)}$ and QB.

\section{B. The sequence $\left[\mathbf{Q B}^{(n)}\right]_{n}$}

The queueing system $\mathbf{Q B} \mathbf{B}^{(n)}$ is the single server infinite capacity queue with deterministic service time equal to one time slot and with input the arrival process $\mathbf{B}^{(n)}$ which is derived from $\mathbf{A}^{(n)}$ in the following way: all customers belonging to a sequence generated by $\mathbf{A}^{(n)}$ enter the buffer simultaneously, i.e. as a batch. The queueing system QB is defined in the same way. It is clear that the processes $\mathbf{B}^{(n)}$ and $\mathbf{B}$ generate the same arrival rate as $\mathbf{A}^{(n)}$ and $\mathbf{A}$, but the covariance structure of the latter two is lost. Denote by $v_{k}^{(n)}$, resp. $v_{k}$, the probability that the buffer in the queueing system $\mathbf{Q B}{ }^{(n)}$, resp. $\mathbf{Q B}$, contains $k$ customers. Hence the generating functions of the stationary buffer occupation distributions are given by $V^{(n)}(z)=\sum_{k=0}^{\infty} v_{k}^{(n)} z^{k}$ and $V(z)=\sum_{k=0}^{\infty} v_{k} z^{k}$.

The following results for these generating functions are well known:

$$
V_{n}(z)=p_{0}^{(n)}(z-1) \frac{B\left(A^{(n)}(z)\right)}{z-B\left(A^{(n)}(z)\right)}
$$

and

$$
V(z)=p_{0}(z-1) \frac{B(A(z))}{z-B(A(z))}
$$

with $p_{0}=1-\rho$. 


\section{Determination of $S(z)$}

A straightforward approach to obtain an expression for $S(z)$ would be to take the limit of $S^{(n)}(z)$, expressed by equation (1), for $n \rightarrow \infty$. However, this method does not lead to a welldefined expression. For example

$$
\prod_{k=1}^{\infty} \frac{B\left(A_{k}(z)\right)}{z}
$$

would diverge on $[0,1)$ because $z<B\left(A_{j}(z)\right)$ for $j \geqslant 1$ and $z \in[0,1)$. Therefor, we transform equation (1). First of all, using the following equality, which holds on some open disk contained in the open unit disk $U(0,1)$ of the complex plane,

$$
\frac{B\left(A_{n}(z)\right)}{z-B\left(A_{n}(z)\right)}=-\sum_{k=0}^{\infty} \frac{z^{k}}{B\left(A_{n}(z)\right)^{k}}
$$

equation (1) can be rewritten as

$$
\begin{gathered}
S_{n}(z)=p_{0}^{(n)}(z-1) \sum_{i=1}^{n-2}\left[\left(\prod_{k=1}^{i} B\left(A_{k}(z)\right)\right) \times\right. \\
\left.\frac{1}{z^{i}}\left(1-\prod_{k=i+1}^{n-1} \frac{B\left(A_{k}(z)\right)}{B\left(A_{n}(z)\right)}\right)\right]+\left[\prod_{k=1}^{n-1} \frac{B\left(A_{k}(z)\right)}{B\left(A_{n}(z)\right)}\right] V_{n}(z) .
\end{gathered}
$$

with the right hand side now representing a holomorphic function on $U(0,1)$.

To keep the notation simple, we introduce the following definitions,

$$
\begin{aligned}
\varphi_{k}^{(n)}(z) & =\sum_{j=k}^{n-1} A_{j}(z)-A_{n}(z), \\
\varphi_{k}(z) & =\sum_{j=k}^{\infty}\left[A_{j}(z)-A(z)\right], \\
\theta_{k}^{(n)}(z) & =\frac{B\left(A_{k}(z)\right) \ldots B\left(A_{n-1}(z)\right)}{B\left(A_{n}(z)\right)^{n-k}}=\exp \left[\lambda \varphi_{k}^{(n)}(z)\right], \\
\theta_{k}(z) & =\exp \left[\lambda \varphi_{k}(z)\right] .
\end{aligned}
$$

Before we are able to proof the main result of this section we need the following lemmas. By $D(0,1)$ we denote the closed unit disk in the complex plane.

Lemma III.1: For each $z \in D(0,1)$ the inequality $\left|B\left(A_{j}(z)\right)\right| \leqslant$ $\exp [\lambda(|z|-1)]$ holds.

Proof: A straightforward calculation using the inequality $A_{j} \leqslant 1$ on $[0,1]$ constitutes the proof.

Lemma III.2: The sequence $\left[\varphi_{k}^{(n)}\right]_{n}$ converges uniformly to $\varphi_{k}$ on $D(0,1)$ for each $k \geqslant 1$.
Proof: Consider $z \in D(0,1)$.

$$
\begin{aligned}
\left|\varphi_{k}(z)-\varphi_{k}^{(n)}(z)\right|=\mid & (n-k)\left[\sum_{k=n+1}^{\infty} a_{k}\right] z^{n}-\sum_{k=n+1}^{\infty} a_{k} z^{k} \\
& +\left[\sum_{k=n+2}^{\infty} a_{k}\right] z^{n+1}-\sum_{k=n+2}^{\infty} a_{k} z^{k} \\
& +\left[\sum_{k=n+3}^{\infty} a_{k}\right] z^{n+2}-\sum_{k=n+3}^{\infty} a_{k} z^{k}+\ldots \mid \\
\leqslant & 2 \sum_{k=n+1} k a_{k} .
\end{aligned}
$$

Hence the uniform convergence is a consequence of $\sum_{k=1}^{\infty} k a_{k}<$ $\infty$.

Lemma III.3: For each $z \in D(0,1)$ the inequalities

$$
\left|\frac{\varphi_{k}^{(n)}(z)}{z^{k-1}}\right| \leqslant 2 \sum_{m>k} m a_{m} \leqslant 2 A^{\prime}(1)
$$

and

$$
\left|\frac{\varphi_{k}(z)}{z^{k-1}}\right| \leqslant 2 \sum_{m>k} m a_{m} \leqslant 2 A^{\prime}(1)
$$

hold for $1 \leqslant k \leqslant n-1$ and $1 \leqslant k<\infty$ respectively.

Proof: Analogous to the proof of lemma III.2.

Lemma III.4: For each $z \in \mathbb{C}$ the inequality $|\exp (z)-1| \leqslant$ $|z| \exp (|z|)$ holds.

Proof: Straightforward.

Now we are able to proof the main result of this section.

Theorem III.1: The generating function $S$ is given by

$$
\begin{array}{r}
S(z)=p_{0}(z-1) \sum_{i=1}^{\infty}\left[\left(\prod_{k=1}^{i} B\left(A_{k}(z)\right)\right) \frac{1}{z^{i}}\left(1-\theta_{i+1}(z)\right)\right]+ \\
\theta_{1}(z) V(z)
\end{array}
$$

Proof: We will proof the pointwise convergence on $[0,1]$ of $S_{n}$ to $S$. By the uniform convergence of $\varphi_{1}^{(n)}$ to $\varphi_{1}$ on [0, $1]$ and by the pointwise convergence of $V_{n}$ to $V$ on [0,1], we immediately obtain,

$$
\lim _{n \rightarrow \infty} \theta_{1}^{(n)}(z) V_{n}(z)=\theta_{1}(z) V(z)
$$

for each $z \in[0,1]$. Furthermore, $S_{n}(1)=S(1)$ and $S_{n}(1)=$ $p_{0}^{(n)}$ which converges to $S(0)=p_{0}$. By these observations we can now restrict our attention to the pointwise convergence of

$T_{n}(z)=p_{0}^{(n)}(z-1) \sum_{i=1}^{n-2}\left[\left(\prod_{k=1}^{i} B\left(A_{k}(z)\right)\right) \frac{1}{z^{i}}\left(1-\theta_{i+1}^{(n)}(z)\right)\right]$

to

$$
T(z)=p_{0}(z-1) \sum_{i=1}^{\infty}\left[\left(\prod_{k=1}^{i} B\left(A_{k}(z)\right)\right) \frac{1}{z^{i}}\left(1-\theta_{i+1}(z)\right)\right]
$$


on $(0,1)$. Let $\epsilon>0$. We will show uniform convergence of $T_{n}$ to $T$ on each interval $(\gamma, 1-\gamma)$ with $0<\gamma<\frac{1}{2}$. Take $z \in(\gamma, 1-\gamma)$. By III.3 we have that

$$
\left|\frac{1}{z^{i}}\left(1-\theta_{i+1}^{(n)}(z)\right)\right| \leqslant 2 \lambda A^{\prime}(1) e^{\left.2 \lambda A^{\prime}(1)\right)}
$$

and

$$
\left|\frac{1}{z^{i}}\left(1-\theta_{i+1}(z)\right)\right| \leqslant 2 \lambda A^{\prime}(1) e^{\left.2 \lambda A^{\prime}(1)\right)}
$$

for $i=1, \ldots, n-2$ and $1 \leqslant i<\infty$ respectively. Hence, by choosing $N$ large enough, one obtains, by using III.1,

$$
\left|p_{0}^{(n)}(z-1) \sum_{i=N}^{n-2}\left[\left(\prod_{k=1}^{i} B\left(A_{k}(z)\right)\right) \frac{1}{z^{i}}\left(1-\theta_{i+1}^{(n)}(z)\right)\right]\right|<\frac{\epsilon}{4}
$$

for each $n>N+2$ and

$$
\left|p_{0}(z-1) \sum_{i=1}^{\infty}\left[\left(\prod_{k=1}^{i} B\left(A_{k}(z)\right)\right) \frac{1}{z^{i}}\left(1-\theta_{i+1}(z)\right)\right]\right|<\frac{\epsilon}{4} .
$$

By the uniform convergence of $\theta_{i+1}^{(n)}$ to $\theta_{i+1}$ on $[0,1]$, a consequence of lemma III.2, and by $p_{0}^{(n)} \rightarrow p_{0}$, it is possible to choose $n$ large enough to obtain

$$
\begin{aligned}
& \left|\left(p_{0}^{(n)}-p_{0}\right)(z-1) \sum_{i=N}^{n-2}\left[\left(\prod_{k=1}^{i} B\left(A_{k}(z)\right)\right) \frac{1}{z^{i}}\left(1-\theta_{i+1}^{(n)}(z)\right)\right]\right| \\
+ & \left|p_{0}(z-1) \sum_{i=1}^{N-1}\left[\frac{1}{z^{i}}\left(\theta_{i+1}(z)-\theta_{i+1}^{(n)}(z)\right) \prod_{k=1}^{i} B\left(A_{k}(z)\right)\right]\right|<\frac{\epsilon}{2} .
\end{aligned}
$$

Hence we have pointwise convergence on $(0,1)$, combined with the convergence at the endpoints, we have $S_{n}(z) \rightarrow S(z)$ for each $z \in[0,1]$.

To simplify forthcoming calculations we rewrite the formula for $S$. The following definitions are used:

$$
\begin{aligned}
\varphi_{k}^{\star}(z) & =A_{1}(z)-A(z)+\ldots+A_{k}(z)-A(z) \\
\theta_{k}^{\star}(z) & =\exp \left[\lambda \varphi_{k}^{\star}(z)\right]
\end{aligned}
$$

Using them enables us to obtain the following result:

$$
\begin{aligned}
S(z)= & p_{0}(z-1) \sum_{i=1}^{\infty}\left[B(A(z))^{i} \theta_{i}^{\star}(z)\left(1-\theta_{i}(z)\right)\right] \\
& +\theta_{1}(z) V(z) \\
= & V(z) \theta_{1}^{\star}(z)+V(z) \sum_{i=1}^{\infty} \theta_{i}^{\star}(z) \frac{B(A(z))^{i}}{z^{i}} \times \\
& {\left[\exp \left(A_{i+1}(z)-A(z)\right)-1\right] . }
\end{aligned}
$$

Again, it is clear that the last formula represents a holomorphic function on $U(0,1)$, and so it is a valid and useful representation of $S(z)$.

\section{THE BUFFER ASYMPTOTICS}

Now we determine the asymptotics of the tail probabilities of the buffer occupancy, i.e. the asymptotic behavior of $\mathbf{P}\left\{X_{S}>\right.$ $n\}=\sum_{k>n}^{\infty} s_{k}$ for $n \rightarrow \infty$. At the same time we obtain the asymptotic behavior of the buffer occupancy for the queueing system QB.

Note that, using the formula

$$
\begin{aligned}
& \mathbf{E}\left[X_{S}\right]=B^{\prime}(1)\left[A^{\prime}(1)-\right.\left.\frac{A^{\prime \prime}(1)}{2}\right]+ \\
& \frac{B^{\prime \prime}(1)\left[A^{\prime}(1)\right]^{2}+B^{\prime}(1) A^{\prime \prime}(1)}{2(1-\rho)}
\end{aligned}
$$

derived in [11], one has

$$
\lim _{z \rightarrow 1-} S^{\prime}(z)=\infty
$$

Determining the exact way in which $S^{\prime}(z)$ diverges for $z \rightarrow 1-$ is the key step in determining the asymptotic behavior of the buffer distribution.

Lemma IV.1:

$$
S^{\prime}(z) \sim \frac{1}{(1-z)^{3-s}} \frac{\lambda c \Gamma(3-s+1) \rho^{s-2}}{(1-\rho)(s-1)(3-s)} \text { for } z \rightarrow 1-
$$

Proof: See appendix.

By the foregoing lemma IV.1 we are able to proof the key result of this paper.

Theorem IV.1: The asymptotic behavior of the tail probabilities of the stationary distribution of the buffer occupancy of the queueing system $\mathbf{Q T}$ is given by

$$
\sum_{k>n} s_{k} \sim \frac{\lambda c \rho^{s-2}}{(s-2)(s-1)(1-\rho)} n^{2-s} \text { for } n \rightarrow \infty .
$$

Proof: By IV.1 and by [13, theorem 5, pg. 423] we have

$$
\sum_{j=1}^{k} j s_{j} \sim \frac{\lambda c \rho^{s-2}}{(1-\rho)(s-1)(3-s)} n^{3-s} .
$$

An application of [14, 3.3 (c), pg. 59] concludes the proof.

From this theorem we conclude that the buffer occupancy distribution has a power-law decay with exponent $2-s$, with $s$ the parameter used in the Pareto distributed length of trains. Besides the slope of the distribution, we also obtain the constant which determines the asymptotic behavior exactly. This asymptotic behavior was postulated, for the fluid flow approach, in [3, theorem 6].

In the same way it is possible to determine the asymptotic behavior for the queueing system QB.

Lemma IV.2:

$$
V^{\prime}(z) \sim \frac{1}{(1-z)^{3-s}} \frac{\lambda c \Gamma(3-s+1)}{(1-\rho)(s-1)(3-s)} \text { for } z \rightarrow 1-
$$

Proof: See appendix.

Theorem IV.2: The asymptotic behavior of the tail probabilities of the stationary distribution of the buffer occupancy of the QB is given by

$$
\sum_{k>n} v_{k} \sim \frac{\lambda c}{(s-2)(s-1)(1-\rho)} n^{2-s} \text { for } n \rightarrow \infty .
$$

Proof: Analogous to the proof of theoremIV.1. 


\section{Application to TrafFic MAnAgEment}

The $\mathrm{M} / \mathrm{G} / \infty$ arrival process can be used as a first approximation when modeling TELNET and FTP sessions. As examined in [6] these sessions arrive according to a Poisson process and their durations/sizes are in several cases consistent with Pareto distributions. Hence, using the asymptotic expression (4) for the buffer occupation, one can now explain the occurrence of the socalled "buffer ineffectiveness" phenomenon. This phenomenon stands for the fact that increasing the buffer sizes beyond a certain value results in only a slight decrease in loss rates when the arriving traffic is LRD, see e.g. [15] and [16]. Clearly, "buffer ineffectiveness" is a consequence of the very slowly decaying tail probabilities given in (4).

In this context we will now study what happens to the buffer asymptotics if we consider:

- the superposition of an SRD and an LRD traffic stream,

- the superposition of two LRD traffic streams.

First of all observe that a superposition of two $\mathrm{M} / \mathrm{G} / \infty$ processes with sequence arrival rates $\lambda_{i}, i=1,2$ and sequence length distributions $\tau_{A_{i}}, i=1,2$, is again a $\mathrm{M} / \mathrm{G} / \infty$ process with $\lambda=$ $\lambda_{1}+\lambda_{2}$ and $\tau_{A}$ determined by $A(z)=\lambda_{1} /\left(\lambda_{1}+\lambda_{2}\right) A_{1}(z)+$ $\lambda_{2} /\left(\lambda_{1}+\lambda_{2}\right) A_{2}(z)$.

\section{A. Superposition of SRD and LRD traffic streams}

Consider an arbitrary distribution with finite variance and generating function $A_{1}(z)$. It is clear that the $\mathrm{M} / \mathrm{G} / \infty$ process, with sequence arrival rate $\lambda_{1}$ constructed from this distribution is SRD. The LRD traffic stream is modeled by an $\mathrm{M} / \mathrm{G} / \infty$ process constructed from $\lambda_{2}$ and $\tau_{A_{2}}$ with $\mathbf{P}\left\{\tau_{A_{2}}=k\right\} \sim c k^{-s}$. It is clear that for the sequence lengths of the superposition one has

$$
\mathbf{P}\left\{\tau_{A}=k\right\} \sim c k^{-s}
$$

Hence

$\mathbf{P}\{Q>k\} \sim \frac{\left(\lambda_{1}+\lambda_{2}\right) c\left(\rho_{1}+\rho_{2}\right)^{s-2}}{(s-2)(s-1)\left(1-\left(\rho_{1}+\rho_{2}\right)\right)} n^{2-s}$ for $n \rightarrow \infty$

with $Q$ representing the buffer occupation and $\rho_{i}$ the loads offered by the individual arrival processes. The contribution of the SRD arrivals is only reflected in the total load and the total arrival rate.

\section{B. Superposition of two LRD traffic streams}

Here both the packet length distributions are Pareto like: $\mathbf{P}\left\{\tau_{A_{i}}=k\right\} \sim c k^{-s_{i}}, i=1,2$. Now

$$
\mathbf{P}\left\{\tau_{A}=k\right\} \sim c k^{-s}
$$

with $s=\min \left(s_{1}, s_{2}\right)$ and $c$ the $c_{i}$ corresponding to this $s$. Again equation (5) describes the asymptotic behavior. As one expects the "worst" behaving source determines the decay of the tail probabilities.

\section{NUMERICAL RESULTS}

\section{A. $M / G / \infty$ simulations}

To get an idea about how fast the asymptotic regime sets in we simulated the "Pareto" queue. For this queue the random variable $\tau_{A}$ is defined by

$$
\mathbf{P}\left\{\tau_{A}=j\right\}=j^{1-s}-(j+1)^{1-s} .
$$

Hence $c=s-1$ and the load $\rho$ is given by $\rho=\lambda \zeta(s-1)$. The random variable $Q$ represents in both examples the stationary buffer occupation distribution.

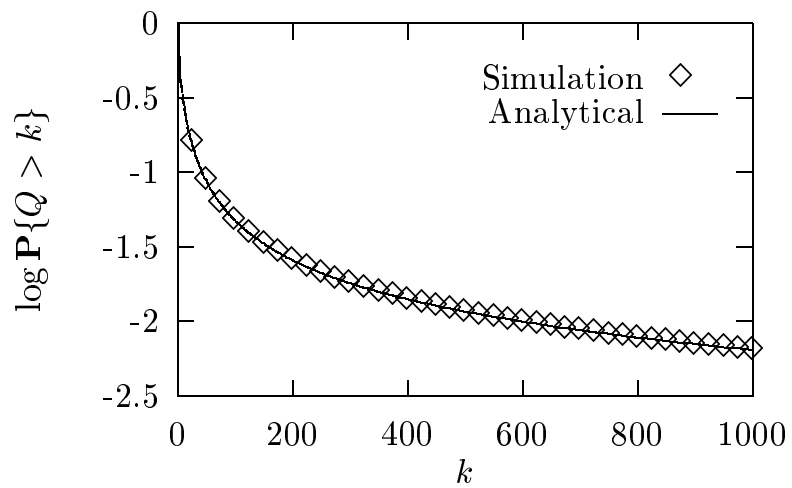

Fig. 1. Example 1

\section{A.1 Example 1}

Here $s=2.8$ and $\lambda=0.4$ resulting in $\rho \approx 0.75$. The simulation length was $10^{9}$ time slots.

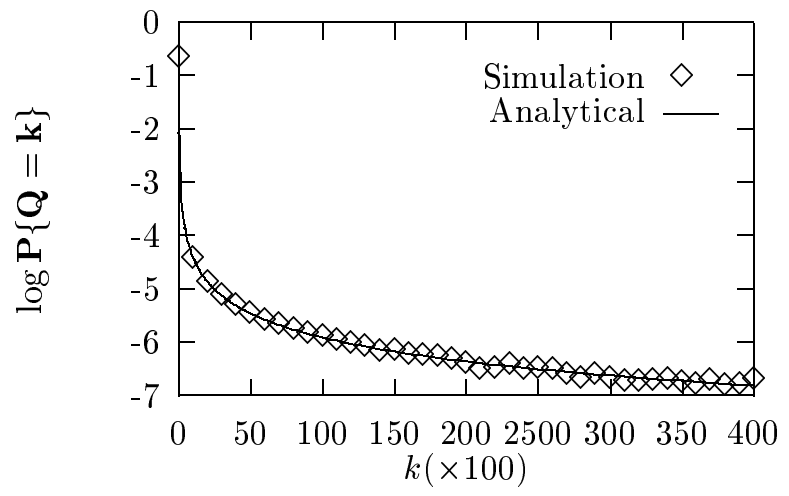

Fig. 2. Example 2

\section{A.2 Example 2}

Here $s=2.5$ and $\lambda=0.2$ resulting in $\rho \approx 0.78$. The simulation length was $2 \times 10^{9}$ time slots. The asymptotic formula for $\mathbf{P}\{Q=k\}$ can be derived from IV.1 and is given by

$$
\mathbf{P}\{Q=k\} \sim \frac{\lambda \rho^{s-2}}{1-\rho} k^{1-s} \text { for } k \rightarrow \infty
$$

As can be seen from the figures, the asymptotic formulas approximate the buffer behavior well over the whole range. 


\section{CONCLUSION}

An exact asymptotic expression describing the tail probabilities of a single server queue with $\mathrm{LRD} M / \mathrm{G} / \infty$ input is derived by using a generating function approach. This result explains the occurrence of the "buffer ineffectiveness" phenomenon observed with LRD traffic streams. As pointed out by the simulation results, the obtained analytical results seem applicable in a more general setting. Therfore future work will focus on generalizing these results.

\section{ACKNOWLEDGMENT}

The authors would like to thank Prof. Jan Van Casteren for the many and helpful discussions.

\section{APPENDIX}

First of all we are going to give the proof of Lemma IV.2, because this lemma will be used in the proof of Lemma IV.1.

Proof of IV.2. Differentiating $V(z)$ and using $B(A(z))=$ $\sum_{k=0}^{\infty}[\lambda(A(z)-1)]^{k} /(k !)$ we obtain

$$
\begin{aligned}
& \lim _{z \rightarrow 1-}(1-z)^{s-3} V^{\prime}(z)= \\
& \lim _{z \rightarrow 1-}(1-z)^{s-3} p_{0} \lambda \frac{1-A(z)-(1-z) A^{\prime}(z)}{[z-B(A(z))]^{2}} .
\end{aligned}
$$

Furthermore,

$$
1-A(z)-(1-z) A^{\prime}(z)=(1-z)^{2} \sum_{k=1}^{\infty} k\left[\sum_{j=k+1}^{\infty} a_{j}\right] z^{j-1} .
$$

Since

$$
k \sum_{j=k+1}^{\infty} a_{j} \sim \frac{c}{s-1} k^{2-s}
$$

for $k \rightarrow \infty$ and

$$
\lim _{z \rightarrow 1-} \frac{(1-z)^{2}}{[z-B(A(z))]^{2}}=\frac{1}{p_{0}^{2}},
$$

we obtain by applying [13, theorem $5, \mathrm{pg}$. 423]

$$
\lim _{z \rightarrow 1-}(1-z)^{s-3} V^{\prime}(z)=\frac{\lambda c \Gamma(3-s+1)}{(1-\rho)(s-1)(3-s)} .
$$

This last equality concludes the proof.

Proof of IV.1. The proof is based on manipulating the expression (3) for $S$. We introduce the following definitions:

$$
\begin{aligned}
\Psi_{i}(z) & =B(A(z))^{i} \theta_{i}^{\star}(z) \frac{1}{z^{i}}\left[\exp \left(A_{i+1}(z)-A(z)\right)-1\right], \\
\Psi(z) & =\sum_{i=1}^{\infty} \Psi_{i}(z) .
\end{aligned}
$$

Hence, we can rewrite (3) as

$$
S(z)=V(z) \exp [\lambda(z-A(z))]+V(z) \Psi(z)
$$

and

$$
\begin{aligned}
& S^{\prime}(z)=V^{\prime}(z) \exp [\lambda(z-A(z))]+ \\
& V(z) \frac{d}{d z} \exp [\lambda(z-A(z))]+V^{\prime}(z) \Psi(z)+V(z) \Psi^{\prime}(z) .
\end{aligned}
$$

First of all we study the behavior of $\Psi(z)$. Each term $\Psi_{i}$ is nonnegative on $[0,1]$ since $A_{j}(z)-A(z) \geqslant 0$ for each $j \geqslant 1$ and for each $z \in[0,1]$. Furthermore, by lemma III.4,

$$
\begin{aligned}
\Psi_{i}(z) & \leqslant \frac{1}{z^{i}} \lambda\left(A_{i+1}(z)-A(z)\right) \exp \left[A_{i+1}(z)-A(z)\right] \\
& \leqslant \frac{1}{z^{i}} \lambda\left(A_{i+1}(z)-A(z)\right) e^{\lambda} \\
& =\left[\left(\sum_{k=n+2}^{\infty} a_{k}\right) z-\sum_{k=n+2}^{\infty} a_{k} z^{k-n}\right] e^{\lambda}
\end{aligned}
$$

$\mathrm{Al}$ this results in

$$
0 \leqslant \Psi(z) \leqslant e^{\lambda}\left[\left(\sum_{k=3}^{\infty}(k-2) a_{k}\right) z-\sum_{k=3}^{\infty}(k-2) a_{k} z^{k}\right] .
$$

Hence,

$$
\lim _{z \rightarrow 1-} \Psi(z)=0 .
$$

Using this result and other more elementary observations we obtain

$$
\begin{aligned}
\lim _{z \rightarrow 1-}(1-z)^{3-s} S^{\prime}(z)=\lim _{z \rightarrow 1-}(1-z)^{3-s} V^{\prime}(z)+ \\
\lim _{z \rightarrow 1-}(1-z)^{3-s} \Psi^{\prime}(z) .
\end{aligned}
$$

Furthermore,

$$
\Psi^{\prime}(z)=\sum_{i=1}^{\infty} \Psi_{i}^{\prime}(z)
$$

because of the uniform convergence of $\left[\sum_{i=1}^{k} \Psi_{i}\right]_{k}$ to $\Psi$ on compact subsets of $U(0,1)$. We will now focus on the behavior of $\Psi^{\prime}(z)$ since we already know the behavior of $V^{\prime}(z)$. Define:

$$
\begin{gathered}
\xi_{i}(z)=\frac{B(A(z))^{i}}{z^{i}} \lambda\left(A_{i+1}(z)-A(z)\right), \\
\omega_{i}(z)=1+\sum_{k=1}^{\infty} \frac{\left[\lambda\left(A_{i+1}(z)-A(z)\right)\right]^{k}}{(k+1) !} .
\end{gathered}
$$

Hence,

$$
\Psi(z)=\sum_{i=1}^{\infty} \theta_{i}^{\star}(z) \xi_{i}(z) \omega_{i}(z)
$$

and

$$
\begin{aligned}
\Psi^{\prime}(z)=\sum_{i=1}^{\infty}\left[\theta_{i}^{\star}(z)\right]^{\prime} \xi_{i}(z) \omega_{i}(z)+ \\
\quad \sum_{i=1}^{\infty} \theta_{i}^{\star}(z) \xi_{i}^{\prime}(z) \omega_{i}(z)+\sum_{i=1}^{\infty} \theta_{i}^{\star}(z) \xi_{i}(z) \omega_{i}^{\prime}(z) .
\end{aligned}
$$


By observing the inequalities

$$
\left[\frac{d}{d z} \theta_{i}^{\star}(z)\right]^{+} \leqslant \lambda \theta_{i}^{\star}(z) \sum_{k=2}^{\infty}(k-1)\left(\sum_{j=k}^{\infty} a_{j}\right) z^{k-2}
$$

and

$$
\left[\frac{d}{d z} \theta_{i}^{\star}(z)\right]^{-} \leqslant \lambda \theta_{i}^{\star}(z) \sum_{k=2}^{\infty} k a_{k} z^{k-1},
$$

with $[f(z)]^{+}=\max \{0, f(z)\},[f(z)]^{-}=-\min \{0, f(z)\}$, one obtains

$$
\lim _{z \rightarrow 1-}(1-z)^{3-s} \sum_{i=1}^{\infty}\left[\theta_{i}^{\star}(z)\right]^{\prime} \xi_{i}(z) \omega_{i}(z)=0 .
$$

Making use of similar techniques one also concludes

$$
\lim _{z \rightarrow 1-}(1-z)^{3-s} \sum_{i=1}^{\infty} \theta_{i}^{\star}(z) \xi_{i}(z) \omega_{i}^{\prime}(z)=0 .
$$

Proceeding in the same way one ultimately obtains

$$
\lim _{z \rightarrow 1-}(1-z)^{3-s} \Psi^{\prime}(z)=\lim _{z \rightarrow 1-}(1-z)^{3-s}\left[\frac{1}{z} \sum_{i=1}^{\infty} \xi_{i}(z)\right]^{\prime} .
$$

Observe that $\frac{1}{z} \sum_{i=1}^{\infty} \xi_{i}(z)$ can be rewritten in a much more tractable way:

$$
\begin{aligned}
\frac{1}{z} \sum_{i=1} \xi_{i}(z)=\lambda & {\left[\sum_{k=1}^{\infty}\left(\sum_{j=k+1}^{\infty} a_{k}\right) B(A(z))^{k}+\right.} \\
& \left.\frac{A(z)-1}{z}+a_{1}-\frac{A[B(A(z))]-A(z)}{B(A(z))-z}\right] .
\end{aligned}
$$

First of all note that

$$
\begin{array}{r}
\lim _{z \rightarrow 1-}(1-z)^{3-s} \lambda\left[\sum_{k=1}^{\infty}\left(\sum_{j=k+1}^{\infty} a_{k}\right) B(A(z))^{k}\right]^{\prime}= \\
\frac{\lambda c \rho^{s-2} \Gamma(3-s+1)}{(s-1)(3-s)} .
\end{array}
$$

Furthermore

$$
\frac{A[B(A(z))]-A(z)}{B(A(z))-z}=\frac{A[B(A(z))]-1}{B(A(z))-z}+\frac{1-A(z)}{B(A(z))-z}
$$

and

$$
\frac{A[B(A(z))]-1}{B(A(z))-z}=v(z) t(z)
$$

with

$$
v(z)=\frac{1-B(A(z))}{B(A(z))-z}
$$

and

$$
t(z)=-\sum_{k=0}^{\infty}\left(\sum_{j>k} a_{j}\right) B(A(z))^{k} .
$$

One has $t(1)=-A^{\prime}(1)$ and

$$
\lim _{z \rightarrow 1-} v(z)=\frac{\rho}{1-\rho} .
$$

Furthermore,

$$
\frac{d}{d z} v(z)=\frac{1-B(A(z))-(1-z)[B(A(z))]^{\prime}}{[B(A(z))-z]^{2}},
$$

and since

$$
\frac{1-A(z)}{B(A(z))-z}=v(z) \frac{1}{\lambda+\frac{\lambda^{2}}{2 !}(A(z)-1)+\ldots},
$$

one obtains

$$
\begin{aligned}
\lim _{z \rightarrow 1-}(1-z)^{3-s} \frac{d}{d z}\left[\lambda \frac{A[B(A(z))]-A(z)}{B(A(z))-z}\right] \\
=\frac{\lambda c \rho^{s-2} \Gamma(3-s+1)}{(s-1)(3-s)}+\frac{\rho}{1-\rho} \frac{\lambda c \rho^{s-2} \Gamma(3-s+1)}{(s-1)(3-s)} \\
\quad-\frac{\lambda c \Gamma(3-s+1)}{(1-\rho)(s-1)(3-s)} .
\end{aligned}
$$

By taking a look at (6) one can now conclude

$$
\lim _{z \rightarrow 1-}(1-z)^{3-s} S^{\prime}(z)=\frac{\lambda c \rho^{s-2} \Gamma(3-s+1)}{(1-\rho)(s-1)(3-s)},
$$

which finishes the proof.

\section{REFERENCES}

[1] W. E. Leland, M. S. Taqqu, W. Willinger, and D. V. Wilson, "On the selfsimilar nature of ethernet traffic (extended version)," IEEE/ACM Transactions on networking, vol. 2, pp. 1-15, february 1994.

[2] M. W. Garret and W. Willinger, "Analysis, modeling and generation of self-similar VBR video traffic," in Proceedings ACM Sigcomm'94, London, UK, 1994, pp. 269-280.

[3] P. R. Jelenković and A. A. Lazar, "Multiplexing on-off sources with subexponential on periods: Part ii," in Teletraffic contributions for the information Age, V. Ramaswami and P.E. Wirth, Eds., Washington D.C., june 1997, ITC, vol. 2b of Teletraffic Science and Engineering, pp. 965-974, Elsevier.

[4] K. Laevens and H. Bruneel, "Some preliminary results on traffic characteristics and queueing behaviour of discrete-time on-off sources," in Proceedings 5th IFIP Workshop on Performance Modelling and Evaluation of ATM Networks, Ilkley, July 1997, pp. 21/1-21/10.

[5] N. Likhanov, B. Tsybakov, and N. D. Georganas, "Analysis of an ATM buffer with self-similar ("fractal") input traffic," in Proceedings of INFOCOM'95. IEEE, 1995.

[6] V. Paxson and S. Floyd, "Wide-area traffic: the failure of poison modeling," in Proc. of the ACM Sigcomm'94, London, UK, 1994, pp. 395-409.

[7] K. P. Tsoukatos and A. M. Makowski, "Heavy traffic analysis for a multiplexer driven by $M / G I / \infty$ input processes," in Teletraffic contributions for the information Age, V. Ramaswami and P.E. Wirth, Eds., Washington D.C., june 1997, ITC, vol. 2a of Teletraffic Science and Engineering, pp. 497-506, Elsevier.

[8] Z. Liu, P. Nain, D. Towsley, and Z. Zhang, "Asymptotic behavior of a multiplexer fed by a long-range dependent process," Tech. Rep. 3230, INRIA, August 1997. 
[9] D. Heath, S. Resnick, and G. Samorodnitsky, "Heavy tails and long range dependence in on/off processes and associated fluid models," Tech. Rep., Cornell University, 1997.

[10] O. J. Boxma, "Fluid queues and regular variation," Tech. Rep., CWI, 1996.

[11] S. Wittevrongel and H. Bruneel, "Queue length and delay for statistical multiplexers with variable-length messages," in Proceedings of GLOBECOM'94, San Fransisco, november 1994, pp. 1080-1084.

[12] S. Wittevrongel and H. Bruneel, "Correlation effects in ATM queues due to data format conversions," Performance Evaluation, , no. 32, pp. 35-56, 1998.

[13] W. Feller, An Introduction to Probability and Its Applications, Volume II, Wiley Series in Probability and Mathematical Statistics. John Wiley \& Sons, Inc., November 1966.

[14] B. M. Makarov, M. G. Goluzina, A. A. Lodkin, and A. N. Podkorytov, Selected Problems in Real Analysis, vol. 107 of Translations of Mathematical Monographs, American Mathematical Society, 1992.

[15] A. Erramilli, O. Narayan, and W. Willinger, "Experimental queueing analysis with long-range depdendent packet traffic," IEEE/ACM Transactions on Networking, vol. 4, no. 2, pp. 209-223, April 1996.

[16] C. L. Hwang and S. Q. Li, "On input state space reduction and buffer noneffective region," in Proc. IEEE Infocom'94, 1994, pp. 1018-1028. 